\title{
Is Stereoscopic 3D a Better Choice for Information Representation in the Car?
}

\author{
Nora Broy \\ BMW Group Research and \\ Technology \\ Hanauerstr. 46 \\ 80992 Munich, Germany \\ nora.nb.broy@bmw.de
}

\author{
Elisabeth André \\ University of Augsburg \\ Universitätsstr. $6 a$ \\ 86159 Augsburg, Germany \\ andre@informatik.uni- \\ augsburg.de
}

\author{
Albrecht Schmidt \\ VIS, University of Suttgart \\ Pfaffenwaldring $5 \mathrm{a}$ \\ 70569 Stuttgart, Germany \\ albrecht.schmidt@vis.uni- \\ stuttgart.de
}

\begin{abstract}
In modern cars users need to interact with safety and comfort functions, driver assistance systems, and infotainment devices. Basic requirements include the perception of the current status and of information items as well as the control of functions. Handling that myriad amount of information while driving requires an appropriate interaction design, structure and visualization of the data. This paper investigates potentials and limitations of stereoscopic 3D for visualizing an in-vehicle information system. We developed a spatial in-car visualization concept that exploits three dimensions for the system's output. Based on a prototype, that implements the central functionality of our concept, we evaluate the 3D representation. A laboratory study with 32 users indicates that stereoscopic 3D is the better choice as it improves the user experience, increases the attractiveness, and helps the user in recognizing the current state of the system. The study shows no significant differences between non-stereoscopic and stereoscopic representations in the users' workload. This indicates that stereoscopic visualizations have no negative impact on the primary driving task.
\end{abstract}

\section{Categories and Subject Descriptors}

H.5.2 [Information Interfaces and Presentation (e.g. HCI)]: User Interfaces — Graphical user interfaces (GUI); Interaction styles (e.g. commands, menus, forms, direct manipulation); Screen design (e.g. text, graphics, color)

\section{Keywords}

3D user interfaces; automotive infotainment; stereoscopic displays

\section{INTRODUCTION}

Although stereoscopy is known over 150 years it never stayed or became mainstream, because of technical difficulties in production and exploitation. In recent years the rapid advancements of stereoscopic digital display technologies, computation power, and signal processing enable high-quality $3 \mathrm{D}$ witnessing acceptance and enthusiasm in the entertainment industry. Beside entertainment related topics, stereoscopic displays find use in vital applications like

This is the author's version of the work. It is posted here for your personal use. Not for redistribution. The definitive Version of Record was published in:

AutomotiveUI'12, October 17-19, Portsmouth, NH, USA.

Copyright (c) 2012 ACM 978-1-4503-1751-1 scientific and medical visualizations of complex three-dimensional objects [12] as well as teleoperation systems for the manipulation of dangerous environments via remotely controlled robots [6].

The perception of depth [25] builds up a mental model about our spatial surrounding by interpreting perceived depth cues. Monocular depth cues are inferred by a single eye and extract spatial information of a sole $2 \mathrm{D}$ view of the scene. Prominent examples are occlusion, perspective, or texture gradients. Binocular cues can only emerge from viewing a scene with both eyes simultaneously. Thereby, the left eye perceives a slightly different image than the right eye. Depending on the use of depth cues, we define three options for presenting visual information: 2D representations use no depth cue, so called $2.5 \mathrm{D}$ projections integrate monocular depth cues, and 3D visualizations additionally apply binocular cues.

The development of interaction, input, and output concepts in the automotive context carry great weight due to the driver's safety related primary task. Although humans use several senses to estimate driving situations the visual perception provides most dominant information. For this reason, the visual output of automotive user interfaces has to be designed properly, allowing the user a fast and intuitive understanding. Particularly in-vehicle information systems (IVIS) make large data volumes accessible to the user. A spatial perception and presentation of the data may contribute to a comprehensible visualization of the information and function variety.

We present a first assessment of the impact of stereoscopic visualizations for user interfaces in the automotive context. We focus on visualizing menu structures of an IVIS. Selected pieces of related work are discussed in Section 2. Assumptions about benefits and risks of stereoscopy for the automotive context (see Section 3 ) form the basis for developing a concept in Section 4 that exploits three dimensions especially for the system output. We propose stereoscopic design guidelines to facilitate developing 3D content of high quality. Section 5 presents the conducted user study with 32 participants comparing a $2.5 \mathrm{D}$ with a $3 \mathrm{D}$ representation of our concept. We discuss future work and give a conclusion in Section 6.

\section{RELATED WORK}

To our knowledge the work by Krüger [14] is the most related to our approach as it provides a theoretical deduction and empirical evaluation of useful applications for 2.5D and 3D displays in vehicles. Krüger focuses on driver assistance systems using the example of adaptive cruise control (ACC). The results show no advantage for stereoscopy in attractiveness and usefulness. Furthermore, the findings of a simulator study evaluating driver distraction indicate that autostereoscopic 3D displays do not influence the driving performance by more frequent and extended glances. How- 
ever, the autostereoscopic display shows significant longer reaction times for distance estimations pertaining the particular use case of ACC. Krüger's approach is strongly related to the ACC use case and the application of a particular autostereoscopic display whose technical state and image quality lacks compared to other available stereoscopic technologies such as shutter. In contrast to the visualization of an ACC system, which consists of only one graphical presentation and does not involve direct interactions, our work focuses on IVISs whose graphical presentation is more comprehensive and interactive. We apply shutter technology which produces high-quality depth impressions since we assume that autostereoscopic technologies are going to achieve a competitive quality in the near future. A detailed overview of the current state of the art regarding stereoscopic display technology offers the survey of Urey et al. [26].

Comparing 2.5D and 3D representations is the purpose of Cours' dissertation, that investigates psychological aspects of perception in combination with $3 \mathrm{D}$ visualizations [5]. It proves the superiority of $3 \mathrm{D}$ against $2.5 \mathrm{D}$ due to increased perceptual performances, lower reaction times, and positive subjective sensitivities. The performance indices are related to a simple experiment which requires the participants to identify the closest object of three side by side depicted spheres. The findings about related subjective sensitivities are gained by displaying image series in $2.5 \mathrm{D}$ and $3 \mathrm{D}$. The $3 \mathrm{D}$ representation causes an increase of positive feelings and a decrease of negative sensitivities with respect to the non-stereoscopic representation.

Alper et al. [1] used stereoscopic depth to highlight regions of interest in graphs by projecting these parts onto a plane closer to the viewpoint of the user. A quantitative study evaluates the effectiveness of stereoscopic highlighting compared to static visual cues (colored concentric circles). The results show no significant difference in user performance between stereoscopic and static visual highlighting. This outcome suggests that stereoscopy has the potential to supplement other highlighting techniques, which can then encode other data attributes. Furthermore, this survey shows that combining stereoscopic and static visual highlighting enhances user performance significantly.

More application-related investigations about the impact of stereoscopy are available in gaming. Mahoney et al. [16] conducted a user study to investigate the subjective experience while playing $3 \mathrm{D}$ games. The results indicate that stereoscopy improves gameplay and immersion if it is applied correctly. The paper stresses that $3 \mathrm{D}$ only shows its advantages if the design and development process focuses on the use of the third dimension and the correct application of stereoscopy. Beside the user study, the work collects some design pointers for developing stereoscopic 3D games. For example, everything should be pushed behind the screen to reduce eye strain and generate the perception of looking into a 3D scene through a window. The survey of Schild and LaViola Jr. [23] examined the impact of stereoscopy on game experience. The results indicate that stereoscopy in games increases experienced immersion, spatial presence, and simulator sickness. Furthermore, results related to attention and cognitive involvement indicate more direct and less thoughtful interactions with stereoscopic games, pointing towards a more natural experience through stereoscopy. In contrast to these investigations, the domain of automotive user interfaces has to consider the primary driving task. We investigate the effectiveness of stereoscopic displays in situations where the users are not able to devote their full attention to the stereoscopic output as it is not the case for games.

Research about user interface concepts for in-car entertainment systems mainly concern interaction techniques, e.g. speech [15],
3D gestures [18], touch [22], and gaze [13]. In contrast, our work mainly focuses on the system's output. Former studies about $2.5 \mathrm{D}$ metaphors for IVIS [4] show that spatial visualizations foster acceptance, usability, and joy of use compared to 2D list based user interface elements. Our work even extends spatial visualization by investigating the impact of $3 \mathrm{D}$ projections.

While this paper investigates the impact of stereoscopic output, current investigations address interacting with stereoscopic visualizations. Hilliges et al. [10] developed HoloDesk, an interactive system combining an optical see through display and Kinect camera to create the illusion that users are directly interacting with 3D graphics. A quantitative user study seems to indicate that stereoscopy can shorten task completion times for direct interactions with augmented reality in comparison to $2.5 \mathrm{D}$. Beside interacting via $3 \mathrm{D}$ gestures with spatial content there are current investigations regarding interaction techniques like touch [27] and gaze [24] in combination with stereoscopic visualizations.

\section{ASSESSING THE IMPACT OF STEREO- SCOPIC DISPLAYS IN THE CAR}

The combination of stereoscopy and an IVIS leads to the assumption that $3 \mathrm{D}$ positively influences the human machine interaction inside a car by increasing joy of use, improving information structuring, and facilitating focus and attention control. But a stereoscopic IVIS also induces the apprehension that stereoscopy implies negative aspects regarding the driver's cognitive and visual workload. 3D may distract the driver from the higher prioritized driving task. These issues raise the question how stereoscopic displays impact the HMI inside the car. We investigate three aspects of in-car stereoscopy, which are presented in more detail by the following.

\subsection{User Experience}

User experience is currently a central topic in the field of Human Machine Interaction. It expresses the feelings and experiences induced in the user's mind while interacting with a system. The term user experience encompasses a variety of meanings ranging from usability to attractiveness and the hedonic, affective use of computational systems (see Hassenzahl and Tractinsky [9] for a cursory sketch of the term user experience). Providing a more natural, intuitive, and immersive experience [23] by enhancing the presentation with a third dimension could positively influence the user's attitude towards a system. Especially entertainment related systems inside the vehicle can benefit from stereoscopic visualizations since attractiveness, joy of use, and appeal are important factors for the automobile industry. Thus, the application of stereoscopy and the interaction with stereoscopic representations might result in a welcome "Wow-effect" on the user's side. This leads to the first hypothesis:

H-ATT: The stereoscopic user interface provides a better user experience and more attractiveness than the non-stereoscopic user interface.

\subsection{Spatial Representation of Information and Structures}

Since the extent of functions and data is still growing in today's IVISs it is necessary to provide clear menu and graphical structures as well as appropriate interaction techniques to allow the driver an easy, intuitive, and fast understanding and handling of the system while driving. Spatial distribution of information might facilitate the design for comprehensible visualizations of huge data volumes. 


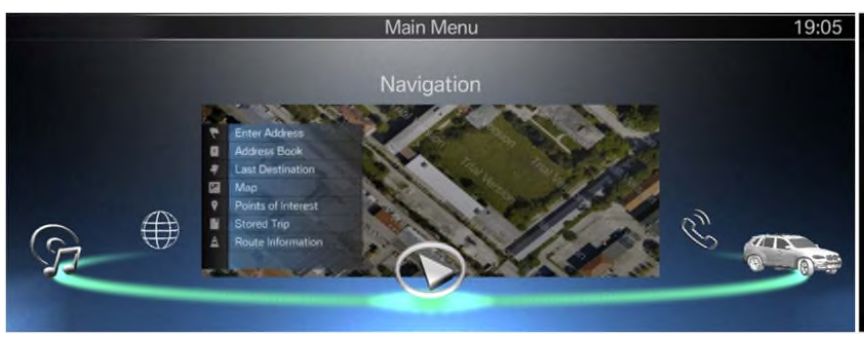

(a) Ring menu.

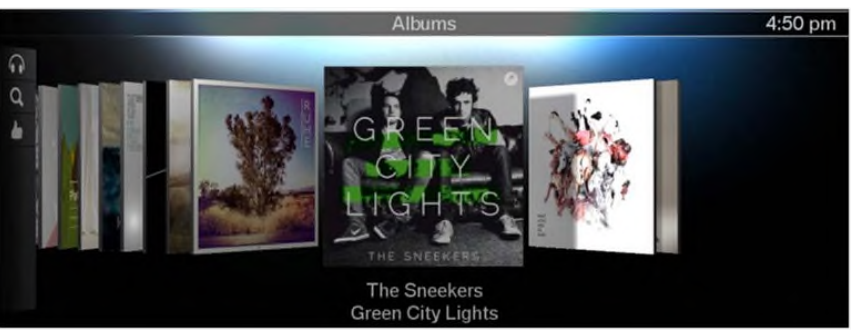

(b) Cover flow.

Figure 1: Examples for spatial elements of the concept.

The following considerations motivate and reason conceptual decisions of Section 4.2. Since the general construction of an IVIS consists of a hierarchical, list-based menu structure it is promising to map this tree-like structure onto spatial visualizations and transitions that are enhanced by comprehensible animations in space [3, 4]. The spatial mapping facilitates to build a cognitive map of the system's content through the avoidance of abstract representations and transitions in the menu tree. This should result in increased learnability and a more intuitive understanding. 3D projections for spatial user interface elements (e.g. cover flow) might profit the user through a better understanding of spatial relations. The introduction of rotary pictorial elements could benefit from the input characteristics of the typical IVIS's rotary input device. Thus, the interaction possibilities allowed by the controller can be directly mapped to the arrangement of the screen's content. A promising aspect of stereoscopy is highlighting [1] and the opportunity to encode additional information via the third dimension. Prioritizing information on the depth axis could emphasize the importance of check controls and warnings. Furthermore, it could be useful to distinguish interaction from information content via depth to indicate with which parts of the screen an interaction may take place and which parts are inaccessible for manipulation. This may help reduce glance times on the screen and enhance safety. The same applies to the differentiation of active and inactive content. Hence, the presentation of the current focus of interaction, meaning the point or the element of the screen's content which is currently controlled, could follow depth values. The depiction of the interaction focus might facilitate the recognition of active elements at present and the current state of the system. Realizing the focus of interaction could make system's reactions to user input more predictable and transparent, so that intended goals are reached immediately. The spatial encoding of the current focus of interaction leads to the following hypothesis:

H-IF: The interaction focus is easier to perceive with the stereoscopic user interface than with the non-stereoscopic user interface.

\subsection{Cognitive and Visual Workload}

Workload concerns the amount of resources that is required to solve a particular task [20]. Cognitive workload refers to cognitive resources and visual workload involves resources that affect visual perception. Visual perception constitutes a part of human cognition. Since binocular vision belongs to visual perception stereoscopy should mainly affect visual workload. Visual fatigue and discomfort may occur from watching stereoscopic content due to conflicting visual cues [21] and 3D of poor quality [17, p.25]. Symptoms like eye strain, headache, dizziness, and tiredness are related to visual fatigue and discomfort. These effects could influence cognitive load. Thus, it is difficult to predict how much stereoscopy affects visual and cognitive workload. Therefore, both terms are not distinguished in this paper. Stereoscopic effects are less serious while playing video games or watching 3D movies because there is no safety-relevant primary task. However, if a car driver suffers from symptoms like headache, dizziness, or motion sickness this could result in critical situations. 3D quality depends on the display technology and the displayed content. An appropriate design of the content and the display reduce and eliminate stereoscopic artifacts and distortions. But in contrast to stereoscopic displays, natural depth perception provides the consistent information from the cues accommodation (monocular) and convergence (binocular) for the visual system. In an artificial 3D environment the viewer's eyes converge to the focused virtual 3D object but the accommodation process sharpens the eyes' lenses on the depth layer of the screen. In consequence, the convergence value varies, while the depth information perceived by accommodation does not change. The rivalry between accommodation and convergence may induce visual fatigue and discomfort. Furthermore, the possibility of encoding more information via the third dimension can also result in information overload. That means the large amount of currently available information hampers the driver in making appropriate decisions or in remaining informed about a topic. Stereoscopy might also lead to perceptual tunneling by distracting the driver in order that the driver becomes focused on a specific stimulus and neglects the primary driving task. Since the perception of stereoscopic content could affect the level of cognitive involvement as well as the visual workload a reduction or loss of the driver's situational awareness is possible. These issues justify the following hypothesis:

H-WL: Interacting with a stereoscopic user interface is accompanied by a higher visual and cognitive workload than handling a non-stereoscopic user interface.

\section{CONCEPT AND IMPLEMENTATION OF A 3D USER INTERFACE}

The user interface development in three dimensions is accompanied by more engineering effort because of guaranteeing a correct use of space, considering 3D object arrangements and consistent spatial transitions. Furthermore, the depth position of each object has to be defined via the stereoscopic parameter parallax. Thereby, zero parallax defines screen depth, positive parallax corresponds to locations behind the display, and objects with negative parallax lie in front of the screen.

\subsection{Design Process}

Our approach for designing a spatial IVIS prototype included three main phases with the aim of creating a well considered 2.5D design which even works in a stereoscopic presentation. In the analysis phase we examined real applications such as IVISs, 2.5D 


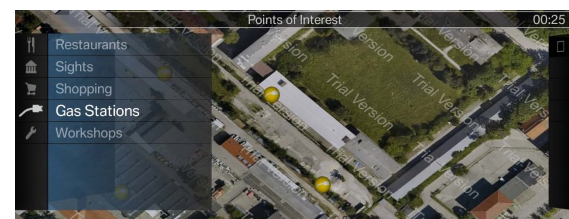

(a) ... menu layer

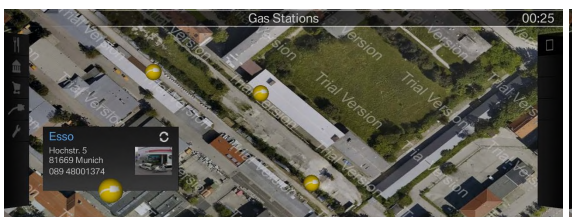

(b) ... application layer

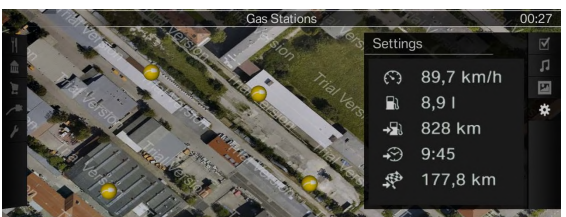

(c) ... splitscreen layer

Figure 2: Focus switch between ...

user interfaces, and stereoscopic applications of the entertainment sector. The analysis' outcomes comprise very basic components of an infotainment system, a collection of well-known 2.5D GUI elements (e.g. ring menu, cover flow), stereoscopic design guidelines, and potential advantages and drawbacks of 3D UIs and stereoscopic displays (see Section 3). In the concept phase we combined these artifacts by matching spatial GUI elements on basic components of an IVIS, maximizing assumed potentials and minimizing possible risks through sticking to proposed stereoscopic guidelines. During the implementation phase we had to take special care of stereoscopic rendering and the used display modality to generate $3 \mathrm{D}$ of high-quality.

Since the quality of stereoscopic visualizations also depends on the displayed content one goal of our work is to collect stereoscopic design guidelines which reduce visual fatigue and discomfort. We identified the following rules through watching 3D movies, playing 3D games, developing this prototype, and studying reviews and interviews with persons responsible for design and production pertaining 3D cinema:

1. Avoid excessive negative parallax.

2. Beware of fast and hectic animations with great parallaxes.

3. Center objects with negative parallax.

4. Avoid presentations with conflicting depth cues ${ }^{1}$.

5. Apply small parallax values to objects of interest.

6. Give only one object or a single group of nearby objects negative parallax at the same time.

7. Only use a small number of depth layers in one screen.

8. Cluster related objects in one depth layer.

9. Use darker colors for the background and lighter colors for the foreground.

10. Avoid high contrasts in color and depth for nearby objects.

These guidelines affect the content design of the user interface instead of stereoscopic display technology to achieve a comfortable $3 \mathrm{D}$ experience. Most rules aim to reduce accommodation convergence conflicts (e.g. 1, 2, 5) and to avoid information overload induced by complex and cluttered visualizations (e.g. 6, 7, 8). Since the proposed guidelines are based on subjective opinions and are not directly evaluated in the conducted user study further research has to clarify if these design rules actually apply.

\footnotetext{
${ }^{1}$ E.g. an object that occludes an object with lower parallax value evokes conflicts between the very strong depth cues binocular disparity and occlusion.
}

\subsection{Concept and UI Design}

The data structures as well as basic elements of an IVIS gain spatial characteristics by applying three-dimensional graphical user interface elements and distributing information in space. For example the main menu is represented by a rotary ring menu, depicted in 1(a), and a cover flow symbolizes a contact or music list (see Figure 1(b)). Lightning, shadowing, and spatial distribution of the elements is well considered to foster three-dimensionality. Moreover, the choice of 3D widgets as well as the spatial organization of the screen's content follow the best possible matching between visual output and user input. The input device is a typical rotary controller commonly used for IVISs. The user can rotate the device in two directions (clockwise and counter-clockwise), press it, or tilt into four directions (up, down, left, right).

The screen is split in three areas for visualizing direct submenus of the main menu. The following points describe the semantics and spatial distribution for each of the three layers:

- The menu layer on the left side of the screen (see Figure 2(a)) consists of panels which contain further submenus. Hence, this layer represents the menu structure of the selected submenu and enables to step through the respective menu hierarchy. Transitions inside the menu layer are visualized by ring file animations between the panels.

- Applications have an increasing relevance for in-vehicle information systems due to the accessibility of online and mobile phone apps. Leaf nodes of the IVIS menu tree are summarized to applications and presented in the application layer in the middle of the screen (note Figure 2(b)). Thus, applications represent subtrees that comprise one or several functions. The currently displayed application is the last one used.

- The user has the possibility to gain further information by enabling a splitscreen. The splitscreen is located at the right side of the screen in the splitscreen layer (note Figure 2(c)). It is possible to choose between several views to configure the displayed information pertaining individual requirements. For instance, the driver may visually follow the guidance of the navigation system and simultaneously gain information about the current played song via an enabled splitscreen (note Figure 3 ). This is beneficial for providing various information without contextual relation at the same time.

Tilting the input device to the left and right enables an immediate focus switch between the introduced layers. This concept shortens transition paths to frequently used functions and to certain menu elements. Furthermore, the consistent spatial distribution allows an intuitive interaction with the system and the detection of fast and simple ways to achieve travel and wayfinding tasks.

Spatial animations should facilitate the understanding of correlations between single data nodes and to remember where to find desired functions or information. Therefore, every transition in the 
menu hierarchy is presented by spatial trajectory movements with the intention to foster the construction of the user's cognitive map.

The third dimension offers apparently an option to encode further information or to emphasize already displayed information. The zooming functionality of the navigation map includes the visual feedback of the currently adjusted scale by indicating the exact number in meters or kilometers. Furthermore, this information is supported by increasing and decreasing the distance between viewer and map over the $\mathrm{z}$-axis. Thus, if the user zooms out of the map it moves away from the viewer. In accordance, zooming in results in a map movement towards the user.

It is also possible to encode meta information about the system and its data via the $\mathrm{z}$-axis. The interaction focus represents the active element of the screen and the system's state. One main constraint of this concept is that exact one element is projected closest to the user's viewpoint. This object defines the current focus of interaction and indicates that proper user input either shifts the focus to another object or manipulates this graphical element. This concept clearly sets the spotlight to active elements and pushes inactive content into the background. Accordingly, the current selected layer is emphasized via depth. Furthermore, toggling between points of interests (POIs) in the navigation map results in pulling the selected POI from the map's positive parallax position to zero parallax (see Figure 3). It is possible to flip selected POIs and covers of the cover flow to their backside in order to access a function list (POI) or music tracks (cover flow). Even the selected list item is highlighted by pushing it towards the viewer.

\subsection{Implementation}

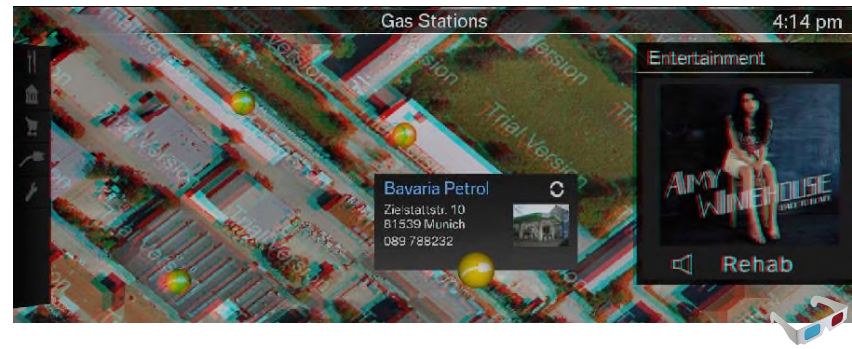

Figure 3: Focused application layer with enabled splitscreen.

A prototype, implemented with the development environment Unity, simulates the front end of the system and enables interaction with main components of the concept. For the simulation of an IVIS without embedding the system in a car the following hardware setup is considered. As input device an iDrive controller [2] is used to provide reasonable control of the system with respect to the automotive context. The controller is connected to a $3 \mathrm{D}$ notebook, which receives the input signals over a CAN bus interface. Since a product of Asus combined with the shutter technology of NVIDIA generates high-quality stereoscopic output [19] the prototype is implemented on an Asus G73JW which is equipped with NVIDIA's 3D Vision kit. This setup allows system output in $3 \mathrm{D}$ and 2.5D and enables immediate switches between both presentation modes. The non-stereoscopic presentation gains threedimensionality through implemented monocular cues (e.g. lighting, shadows, linear perspective, ...). In addition to the realized monocular cues, the stereoscopic visualization involves binocular disparity. Figure 3 shows an anaglyph screenshot and consequently a $3 \mathrm{D}$ representation of the developed prototype. The generation of 3D output with NVIDIA's solution basically depends on two parameters which we adjusted in accordance to the in-car situation and NVIDIA's recommendations [7].

\section{EVALUATION}

We conducted a laboratory study with 32 participants to evaluate the differences between interacting with a $2.5 \mathrm{D}$ and $3 \mathrm{D}$ representation of the prototype. The goal of our experiment is to examine the hypotheses of Section 3 about attractiveness, interaction focus, and workload. Our study does not include a primary driving task.

\subsection{Applied Methods}

The experiment involved objective as well as subjective measures, which are outlined in this section.

\subsubsection{Objective Methods}

The Peripheral Detection Task (PDT) is applied in this laboratory survey to evaluate hypothesis H-WL. The PDT and some variations of it have already been applied to field, simulator, and laboratory studies in recent years to assess driver distraction as well as visual and cognitive workload caused by an IVIS [11]. The standard PDT requires simple manual responses on light stimuli, which are presented in the peripheral vision of the participant. The number of detections and the reaction time on presented stimuli represent useful performance indices for measuring variations in workload. For our approach the light stimuli of the PDT are randomly presented at five possible positions on a black TV flat screen. Every stimulus is shown for maximal one second. If the user does not react by hitting the spacebar on a keyboard within 2 seconds from the onset of the stimulus it is counted as a missed signal. The time interval between successive stimuli is randomized between 3 and 5 seconds.

The Total Task Time (TTT) measures the elapsed time by completing a given task in the system. Since a higher cognitive and visual workload could affect the performance of completing a certain task this value should validate the results of the PDT. Accordingly, the TTT is related to hypothesis H-WL.

\subsubsection{Subjective Methods}

A subset of the standardized 21 items AttrakDiff questionnaire [8] is used to test hypothesis HQ-ATT. This small version of the AttrakDiff consists of 10 adjective pairs and is suitable for repeated assessments during a test session. Adjective pairs are semantic differentials rated on a seven point Likert scale. The items are grouped into three dimensions. Four items (e.g. impractical - practical) cover pragmatic quality (PQ) representing the subjectively perceived usability of the system. Hedonic quality (HQ), addressed by four items as well (e.g. tacky - stylish), describes on the one hand to what extent the system supports the human need to develop and move forward and on the other hand to what extent the user identifies with the system. The third dimension is covered by two items (e.g. bad - good) describing the overall attractiveness (ATT). Two more adjective pairs (ordinary - novel and disagreeable - likable) are added from the original 21 items AttrakDiff since these items appear to be promising in showing significant differences between a $2.5 \mathrm{D}$ and $3 \mathrm{D}$ user interface.

To validate the results of the AttrakDiff a comparison questionnaire is designed containing nine items that directly retrieve the preference of $2.5 \mathrm{D}$ or $3 \mathrm{D}$ on a two point Likert scale. The nine items rate the preference pertaining the general impression, animations, and seven concrete elements of the system (e.g. ring menu, cover flow, list selection, ... ). Furthermore, this questionnaire integrates three use cases (switching between the three layers; toggling between POIs; list selection) rated on a seven point Likert scale to evaluate if the current interaction focus is easier to perceive with a $2.5 \mathrm{D}$ or $3 \mathrm{D}$ representation. The comparison questionnaire should prove hypotheses H-ATT and H-IF. 
The test person answers two direct questions for different use cases (toggling between POIs; switching between the three layers) while interacting with the system in $2.5 \mathrm{D}$ resp. $3 \mathrm{D}$ mode. The participant rates from a ten point Likert scale how easy it is to recognize the current interaction focus even while averting the eyes from the prototype and focusing on it again. These questions test hypothesis H-IF and validate the result of the comparison questionnaire.

The Rating Scale Mental Effort (RSME) [28] is a subjective method for measuring workload. It consists of a 150-point scale on which the participant marks the perceived effort in completing a certain task. The RSME is related to hypothesis H-WL as subjective measurement to validate the results of the PDT and TTT.

\subsection{Study Design}

We used a within groups study design, meaning every participant was confronted with the stereoscopic and non-stereoscopic representation. There were two groups which differed in the order of experiencing the $2.5 \mathrm{D}$ or $3 \mathrm{D}$ presentation to avoid learning and fatigue effects due to the presentation sequence. Consequently, group A always experienced the non-stereoscopic version before the stereoscopic representation and vice versa for group B. The division of the test persons to the groups happened randomly. The two groups had equal sample sizes, gender ratios, and approximately the same average age.

In this study the independent variable is the representation mode of the developed prototype which is either stereoscopic or nonstereoscopic. This is the only variable which is altered during the study influencing a number of dependent variables that measure attractiveness, interaction focus, and workload (see Section 5.1).

\subsection{Procedure and Experimental Setup}

The procedure consists of three parts and is introduced exemplary for group A. In the first part the participant is welcomed by a short explanation of stereoscopy and a brief overview of the session. A stereo vision test takes place to assure that the test person is able to perceive stereoscopic content. If the participant does not pass this test the session ends at this point. Fortunately, all 32 participants qualified for further parts of the evaluation.

The second part starts with exploring the system in 2.5D mode for group A. The exploration is half guided to ensure that the test person becomes acquainted with every detail of the system. The participant has to answer the two direct questions after the related use cases are discovered. After the exploration, the test person completes the AttrakDiff for the 2.5D presentation. Then the whole process, including the exploration, the two direct questions, and the completion of the AttrakDiff, is repeated but this time in 3D mode. Finally, this part ends by completing the comparison questionnaire.

The last part of the evaluation concerns the visual and cognitive workload. The PDT occurs on a TV screen, which is directly placed in front of the participant. Figure 4 shows the 3D notebook with the running prototype and the input controller, placed to the participants' right, and a keyboard for reactions on PDT stimuli to their left. A yellow cross on the TV screen helps finding similar positions for all test persons. At first, a baseline run of the PDT takes place. The participant is instructed to focus the yellow cross and to react immediately on appearing light stimuli by pushing the spacebar of the PDT keyboard. After practicing given tasks (see Table 1) within the IVIS system, the participant has to complete two task sequences (T1-T2-T3 and T3-T2-T1) as fast as possible (TTT recording) while reacting simultaneously to PDT stimuli. This part occurs once with the $2.5 \mathrm{D}$ and once with the $3 \mathrm{D}$ representation. The participant is instructed to give the IVIS tasks a higher priority

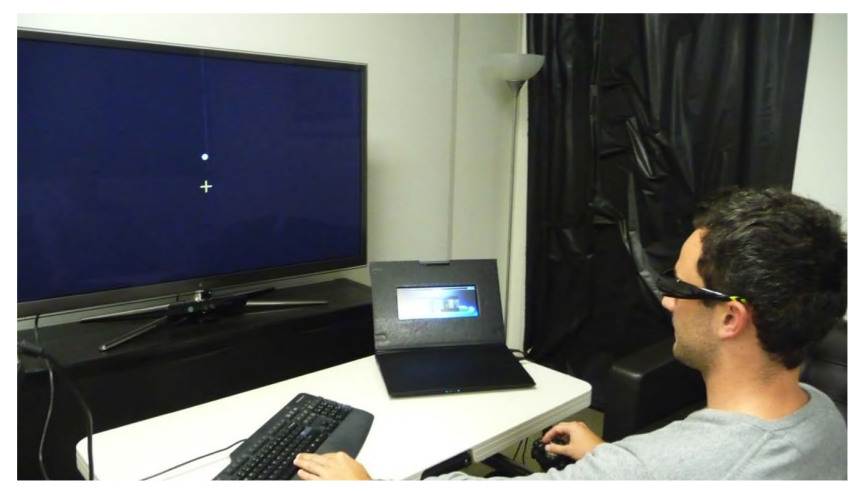

Figure 4: Participant completing a task within the IVIS prototype accompanied by the PDT.

but with the intention to finish both tasks as accurately as possible. After each run of the PDT (baseline, 2.5D, 3D) the participant rates the perceived effort by completing the RSME.

One session lasts for approximately one hour. The test person has to wear the 3D glasses during the whole experiment, even if the presentation is in glasses-free 2.5D. This should prevent inadvertent effects and result in assessments independent of the glasses.

\begin{tabular}{|c|l|}
\hline T1: & $\begin{array}{l}\text { Selecting the song "Brain Damage" of Pink Floyd in the } \\
\text { cover flow }\end{array}$ \\
\hline T2: & $\begin{array}{l}\text { Starting the navigation to the "Esso" gas station via the } \\
\text { POI selection }\end{array}$ \\
\hline T3: & Enabling the splitscreen on the view "Settings" \\
\hline
\end{tabular}

Table 1: IVIS Tasks accompanied by the PDT.

\subsection{Presentation and Discussion of Results}

In the following the results of the conducted user study are presented and discussed. A significance level of 5\% is determined for the applied tests.

\subsubsection{Attractiveness}

The used AttrakDiff shows significant differences for the dimensions HQ $\left(t_{(31)}=-6.255, p<0.001\right)$ and ATT $\left(t_{(31)}=-4.257\right.$, $p<0.001)$. The two additional chosen AttrakDiff items reinforce these findings since novel - ordinary $\left(t_{(31)}=-8.190\right.$, $p<0.001)$ belongs to HQ and likable - disagreeable $\left(t_{(31)}=\right.$ $-2.572, p=0.015)$ is part of the dimension ATT. In contrast, there are no significant differences regarding PQ $\left(t_{(31)}=-1.049, p=\right.$ $0.302)$. We assume that this dimension is rated similar since the overall functionality and controlling of the evaluated variants is exactly the same for the $2.5 \mathrm{D}$ and $3 \mathrm{D}$ system. Nevertheless, the stereoscopic user interface increases hedonic quality and attractiveness. The comparison questionnaire supports this finding since a predominant majority prefers the $3 \mathrm{D}$ version regarding the entire system (25 preferred 3D), its animations (28 preferred 3D), and its graphical components. Only the list selection does not show a very strong majority (20 preferred 3D). We assume that this result emanates from the list selection on the backside of the POIs and the covers in the cover flow because these use cases apply extreme negative parallaxes. Four participants described their negative experiences with 3D as follows: "Stereoscopic displays make me dizzy.", "I find a noticeable difference in focusing effort between the two modes. While the stereoscopic mode is definitely more captivating, I still get a lot of pleasure of the high-quality animation and rendering of the non-stereoscopic display", "There seemed to be 
several levels of depth and it was a bit more difficult to distinguish those.", "[...] stereoscopy makes my eyes tired after extended use.". In contrast, especially the cover flow (29 preferred 3D), the focus switch between menu, application, and splitscreen layer (30 preferred 3D), and toggling between POIs (30 preferred 3D) show very high majorities on the $3 \mathrm{D}$ side. The participants generally justified their preference towards the stereoscopic presentation with "having more fun", "stereoscopic presentation is simply more progressive and interesting", "stylish", "the ease of 3D presentation in finding icons and navigation through screens", "easier to handle", "more intuitive", providing "a better feeling of the system", and "a better understanding of the UI design". Consequently, the applied methods support hypothesis ATT. In contrast to Krüger's work [14] but in accordance to Cours findings [5], our study suggests that stereoscopy increases attractiveness and positive sensitivities. Overall, the generally positive results of the AttrakDiff (see Figure 5) reflect the suitability of the developed concept.

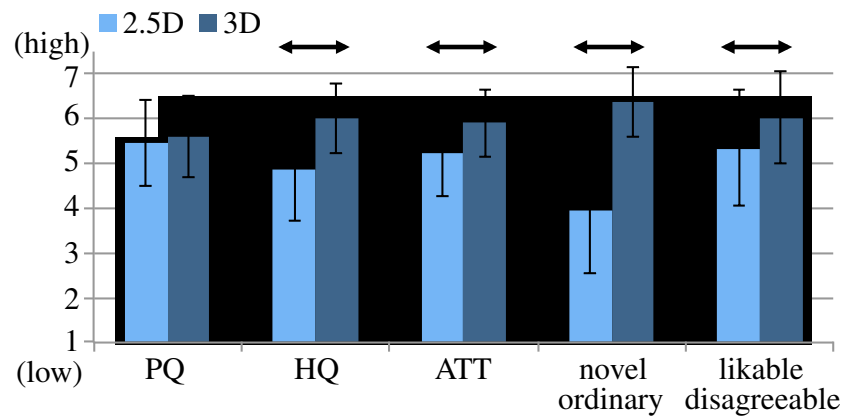

Figure 5: Means and standard deviations of the AttrakDiff dimensions, arrows indicate significant differences.

\subsubsection{Focus Control}

The participants clearly rated that the interaction focus is easier to perceive with the 3D representation of the UI for all prompted use cases in the Comparison Questionnaire. The test persons justified their decisions as follows: "Visually understanding what was active, and what I was doing to the system was much more natural.", "Focused elements were a lot easy to identity in the stereoscopic version in comparison to the non-stereoscopic.", "Generally focus changes are clearer and complex information can be more easy conveyed.", "The stereoscopic views have the added depth information that make it more clear about what you should be focusing on.". The results of the direct questions support this outcome. Both evaluated use cases show extreme significance levels $\left(\left(t_{(31)}=7.098, p<0.001\right),\left(t_{(31)}=5.861, p<0.001\right)\right)$ for the benefit of the stereoscopic version of the UI. Due to these findings it is easier to perceive the current focus of interaction with the $3 \mathrm{D}$ representation, supporting hypothesis H-IF. These subjective findings emphasize the potential of stereoscopic highlighting and the quantitative results of Alper et al. [1]. However, the 2.5D variant only uses monocular depth cues for highlighting. Comparing stereoscopic highlighting for the current focus of interaction to other highlighting methods as color provides an interesting field for further research.

\subsubsection{Workload}

Figure 6 illustrates the results of the PDT indices. A one-way ANOVA shows that there are highly significant differences between the three datasets baseline, $2.5 \mathrm{D}$, and 3D regarding the PDT performances detection rate $\left(F_{(2,62)}=11.057, p<0.001\right)$ and the reaction time $\left(F_{(2,62)}=145.038, p<0.001\right)$. The results of a Tukey's HSD (Honestly Significant Difference) test (see Table 2) show which means are significantly different from one another. Between the $2.5 \mathrm{D}$ and $3 \mathrm{D}$ system no significant difference is given regarding detection rate as well as reaction time. However, the baseline shows significant differences to both system variants regarding reaction time and detection rate. This indicates that the used setup of the PDT is appropriate for measuring the workload since the obvious workload difference between accomplishing only the PDT and in relation with a simultaneous task is ascertained.

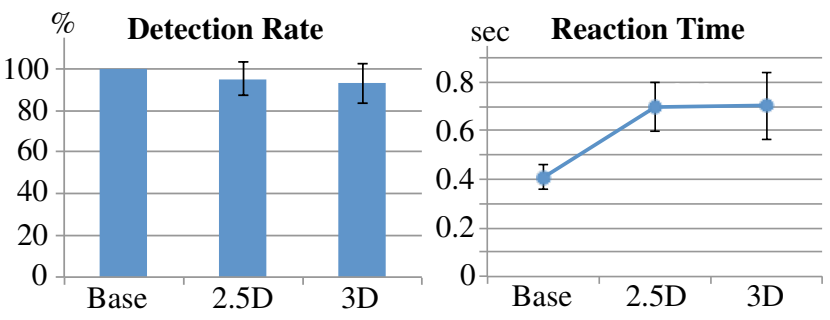

Figure 6: Results of the PDT.

\begin{tabular}{|l|c|c|c|}
\hline \hline \multicolumn{4}{|c|}{ PDT \& RSME } \\
\hline \hline Measurement: & Detection Rate & Reaction Time & RSME \\
\hline 2.5D vs. 3D: & $\mathrm{p}=0.253$ & $\mathrm{p}=0.98$ & $\mathrm{p}=0.948$ \\
\hline Base vs. 2.5D: & $\mathrm{p}=0.0067$ & $\mathrm{p}<0.001$ & $\mathrm{p}<0.001$ \\
\hline Base vs. 3D: & $\mathrm{p}<0.001$ & $\mathrm{p}<0.001$ & $\mathrm{p}<0.001$ \\
\hline
\end{tabular}

Table 2: P-values of Tukey's range test regarding the PDT performance indices and the subjective RSME ratings.

Since the PDT can differ the workload of completing the PDT alone or accompanied by tasks in the system it proves its validity. But its sensitivity is unknown. However, the results of the TTT and the subjective ratings of the RSME validate the findings of the PDT. The outcomes of the TTT do not exhibit a performance difference among task completion with a $2.5 \mathrm{D}$ and 3D user interface $\left(t_{(31)}=\right.$ $-1.583, p=0.124)$. The subjective ratings of the RSME exactly reflect the results of the PDT. The RSME results are statistically analyzed by a one-way ANOVA $\left(F_{(2,62)}=89.334, p<0.001\right)$ and a post hoc Tukey's range test (see Table 2 ).

Our experiment does not show significant differences in the user's workload between a $2.5 \mathrm{D}$ and $3 \mathrm{D}$ visualization for the applied subjective (RSME) and objective methods (PDT, TTT). Hence, our approach does not allow the acceptance of hypothesis H-WL. When viewed together with Krüger's results about the impact of stereoscopy on driving distraction [14], our findings indicate that 3D does not negatively influence the primary driving task.

\section{CONCLUSIONS AND FUTURE WORK}

Our research shows that stereoscopic 3D representations are a valid and promising approach for in-car user interfaces. The results from our study provides evidence that $3 \mathrm{D}$ representations have a potential to improve the user experience and the attractiveness of user interfaces without a negative impact on the user's workload.

In general, developing user interfaces with stereoscopic output requires more effort because of designing an appropriate spatial UI concept, the graphical implementation as well as the selection and integration of a 3D display technology. However, the increased effort is worthwhile as it enhances the perceived quality of user interface of IVIS systems as well as joy of use. Furthermore, our research indicates that the well-considered use of space combined with a stereoscopic presentation contributes to the comprehensibility of the system. The structuring of the screen's content in a way that the currently focused element is the foremost object helps the 
user intuitively recognize the current focus of interaction and consequently the state of the system. Additionally, our findings indicate that stereoscopy has no negative impact on the driver's workload, since we could not find significant differences between a 2.5D and $3 \mathrm{D}$ representation in visual and cognitive workload. Hence, our assumption is that stereoscopic displays do not increase distraction from the primary driving task. We plan to investigate this in more detail since our study does not involve the primary driving task. We argue that laboratory and field studies, including the actual driving task, have to validate and confirm our findings.

We assume that 3D quality is crucial for stereoscopy to show up its advantages and for eliminating possible drawbacks. Our approach integrates shutter technology since it provides the best stereoscopic experience. However, the installation of stereoscopic displays in the car needs autostereoscopic technologies since the driver should not be distracted by glasses. Indeed, the development of autostereoscopic technologies is in progress [26] and it is conceivable that in a few years autostereoscopic displays achieve a 3D quality that is comparable to other stereoscopic technologies. Beside display technology, 3D quality is dependent on its visualized content. We collected design guidelines for stereoscopic user interfaces to facilitate 3D content design. In the next step user studies have to extend, validate, and concretize the proposed guidelines.

Finally, our approach focuses more on output and less on input. Current investigations in the field of user interfaces visualized by stereoscopic technologies concentrate on user input. Future research should identify appropriate interaction techniques.

\section{ACKNOWLEDGMENTS}

This work was created during the course of the Software Engineering Elite Graduate Program and in cooperation with the BMW Technology Office in Mountain View, CA. We would like to thank BMW Group Research and Technology and the Software Engineering Graduate Program for their kind support.

\section{REFERENCES}

[1] B. Alper, T. Höllerer, J. Kuchera-Morin, and A. Forbes. Stereoscopic highlighting: 2d graph visualization on stereo displays. IEEE Trans. Vis. Comput. Graph., 17(12):2325-2333, 2011.

[2] BMW Techniklexikon. iDrive Controller. http://www.bmw.com/com/en/insights/technology/ technology_guide/articles/controller.html.

[3] V. Broy. Benutzerzentrierte, graphische Interaktionsmetaphern für Fahrerinformationssysteme. $\mathrm{PhD}$ thesis, Technische Universität München, 2007.

[4] V. Broy, F. Althoff, and G. Klinker. iflip: a metaphor for in-vehicle information systems. In Proc. AVI '06, pages 155-158. ACM Press, 2006.

[5] N. Cours. Wahrnehmungspsychologische Evaluation eines dreidimensionalen Visualisierungssystems. $\mathrm{PhD}$ thesis, Universität Kassel, 2004.

[6] M. Ferre, R. Aracil, and M. A. Sánchez-Urán. Stereoscopic Human Interfaces. IEEE Robotics \& Automation Magazine, 15(4):50-57, December 2008.

[7] S. Gateau and R. Neuman. Stereoscopy From XY to Z. In Courses of SIGGRAPH Asia 2010, 2010.

[8] M. Hassenzahl and A. Monk. The Inference of Perceived Usability From Beauty. Human-Computer Interaction, 25(3):235-260, 2010.

[9] M. Hassenzahl and N. Tractinsky. User experience - a research agenda. Behaviour \& Information Technology,
25(2):91-97, 2006.

[10] O. Hilliges, D. Kim, S. Izadi, M. Weiss, and A. Wilson. Holodesk: direct 3d interactions with a situated see-through display. In Proc. CHI '12, pages 2421-30. ACM Press, 2012.

[11] G. Jahn, A. Oehme, J. F. Krems, and C. Gelau. Peripheral detection as a workload measure in driving: Effects of traffic complexity and route guidance system use in a driving study. Transportation Research Part F: Traffic Psychology and Behaviour, 8:255-275, 2005.

[12] N. W. John. Using stereoscopy for medical virtual reality. volume 85 of Studies in health technology and informatics, pages 214-220. IOS Press, 2002.

[13] D. Kern, A. Mahr, S. Castronovo, A. Schmidt, and C. Müller. Making use of drivers' glances onto the screen for explicit gaze-based interaction. In Proc. AutomotiveUI '10, pages 110-113. ACM Press, 2010.

[14] K. Krüger. Nutzen und Grenzen von 3D-Anzeigen in Fahrzeugen. $\mathrm{PhD}$ thesis, Humboldt-Universität zu Berlin, 2007.

[15] A. L. Kun, A. Shyrokov, and P. A. Heeman. Spoken tasks for human-human experiments: towards in-car speech user interfaces for multi-threaded dialogue. In Proc. AutomotiveUI '10. ACM Press, November 2010.

[16] N. Mahoney, A. Oikonomou, and D. Wilson. Stereoscopic 3D In Video Games: A Review Of Current Design Practices And Challenges. In Proc. CGAMES '11, pages 148-155. IEEE, 2011.

[17] B. Mendiburu. 3D Movie Making: Stereoscopic Digital Cinema from Script to Screen. Elsevier, 2009.

[18] C. Müller and G. Weinberg. Multimodal Input in the Car, Today and Tomorrow. IEEE Multimedia, 18:98-103, 2011.

[19] F. Müssig. Mobiles mit 3D. c't, pages 102-109, September 2011.

[20] R. D. O'Donnell and F. T. Eggemeier. Workload assessment methodology. Handbook of perception and performance: Cognitive processes and performance, II:42/1-42/49, 1986.

[21] R. Patterson and A. Silzars. Immersive stereo displays, intuitive reasoning, and cognitive engineering. In Stereoscopic Displays and Virtual Reality Systems X, volume 17, pages 443-448, 2009.

[22] H. Richter, R. Ecker, C. Deisler, and A. M. Butz. HapTouch and the 2+1 State Model: Potentials of Haptic Feedback on Touch Based In-Vehicle Information Systems. In Proc. AutomotiveUI '10, pages 72-79. ACM Press, 2010.

[23] J. Schild, J. LaViola, and M. Masuch. Understanding user experience in stereoscopic 3d games. In Proc. CHI' 12, pages 89-98. ACM Press, 2012.

[24] S. Stellmach and R. Dachselt. Looking at 3d user interfaces. In CHI '12 Workshop on The 3rd Dimension of CHI (3DCHI), 2012.

[25] R. J. Sternberg, J. Mio, and J. S. Mio. Cognitive psychology. Cengage Learning, 5, illustrated edition, 2009.

[26] H. Urey, K. V. Chellappan, E. Erden, and P. Surman. State of the Art in Stereoscopic and Autostereoscopic Displays. Proceedings of the IEEE, 99(4):540-555, April 2011.

[27] D. Valkov, F. Steinicke, G. Bruder, and K. Hinrichs. 2d touching of 3d stereoscopic objects. In Proc. CHI'11, pages 1353-1362. ACM Press, 2011.

[28] F. R. H. Zijlstra. Efficiency in work behaviour. A design approach for modern tools. $\mathrm{PhD}$ thesis, Technische Universiteit Delft, 1993. 